Title : T lymphocyte-based renin angiotensin system in obesity.

Running Title : T-cell renin angiotensin system in obesity

\title{
Authors :
}

Mirella Coppo *\# BSc, Manuela Bandinelli *\# BSc, Marco Chiostri *० MD, Loredana Poggesi *० MD, Maria Boddi *o MD

*Affiliation: Department of Experimental and Clinical Medicine, University of Florence, Florence, Italy.

\section{Corresponding Author:}

Mirella Coppo, BSc

Department of Experimental and Clinical Medicine,

University of Florence, Largo Brambilla 3, 50134 Florence, Italy.

Tel.: $00390557947007 \quad$ Fax : 00390557947284

E-mail :mirella.coppo@unifi.it

Funding: This research received no grant

Declaration of Conflicting Interests: The authors have no conflict of interest to declare. 


\section{Background}

Obesity can be associated with increased cardio-metabolic risk, but some subjects with obesity do not show metabolic impairment and escape this association. Low grade inflammation (i.e., high sensitivity C-reactive protein [hsCRP] $>3 \mathrm{mg} / \mathrm{dl}$ ) is associated with high cardiovascular risk in obesity. We investigated renin-angiotensin system (RAS) activity in cultured circulating T-cell in subjects with obesity with and without angiotensin II (Ang II) stimulation in the presence or absence of low-grade inflammation.

\section{Materials and methods}

We studied 18 subjects with obesity and 10 healthy subjects. After T-lymphocyte isolation, Tcells mRNAs for angiotensin converting enzyme (ACE) and AT1-receptor were quantified by reverse transcription polymerase chain reaction at baseline and after Ang II stimulation. hsCRP, plasma renin and ACE activity in the cell pellet and supernatant and Ang II T-cell content were also measured.

\section{Results}

T-cell RAS in subjects with obesity with low grade inflammation was more activated than in subjects with obesity without low grade inflammation. The increase in RAS activation occurred both at baseline and after Ang II stimulation. Similarly the release of ACE activity in the supernatant was significantly higher in subjects with obesity with hsCRP>3 $\mathrm{mg} / \mathrm{dl}$ tha in subjects hsCRP $<3 \mathrm{mg} / \mathrm{dl}$ and controls.

\section{Conclusions}

Circulating T-cell based RAS is activated in subjects with obesity independently of low grade inflammation that amplifies the T-cell RAS response to Ang II stimulation. 
Keywords : Angiotensin-converting enzyme; Angiotensin II ; T-cell; Obesity; Renin-angiotensin system.

\section{INTRODUCTION}

Obesity is a complex disease characterized by excessive expansion of adipose tissue and is an important risk factor for chronic diseases such as cardiovascular disorders, hypertension and type 2 diabetes ${ }^{1}$ Accumulated evidence suggests that the renin-angiotensin system (RAS) strictly relates to inflammation, mitochondrial dysfunction and oxidative stress as possible mechanisms linking RAS to obesity and insulin resistance. ${ }^{2}$

Obesity is characterized by chronic low-grade systemic inflammation, which in part emanates from adipose tissue inflammation. This occurs when immune cells such as macrophages and Tcells infiltrate adipose tissues ${ }^{3}$ and secrete pro-inflammatory substances including Angiotensin II (Ang II), that spill into the bloodstream and contribute to systemic inflammation.

Adipose tissue has been considered recently as a distinct endocrine organ that produces multiple bioactive mediators, known as adipokines that play a functional role in the regulation of metabolism, inflammation and tissue remodeling . ${ }^{4}$ Recent studies indicate that a functional RAS exists in the adipose tissue and is involved in the pathophysiology of metabolic disorders. ${ }^{5}$ Adipose tissue RAS overactivation causes glucose intolerance and systemic insulin resistance. ${ }^{6}$ Accordingly both adipose-specific gene overexpression and knockout mouse models of RAS components were investigated. ${ }^{7,8,9}$ Over-expression of angiotensinogen (Agt) specifically in adipose tissue resulted in increased fat mass, fat cell size, adipose and systemic inflammation as well as insulin resistance. By contrast, inactivation of Agt in adipose tissue improved glucose clearance, reduced inflammation, adipose tissue macrophage infiltration and also enhanced metabolic activity.$^{10,11,12}$ 
The pathophysiological actions of Ang II are mediated by the angiotensin II type 1 receptor (AT1R). Therapeutic effects of irbesartan, an AT1R specific blocker, were recently shown in genetically obese diabetic KKAy mice, a model of human metabolic disorders without any dietary loading, with focus on the analysis of possible effects of irbesartan on adipose tissue. The treatment with irbesartan significantly lowered systolic blood pressure with a concomitant decrease in body weight in KKAy mice.$^{13}$

Human visceral adipose tissue is rich in lymphocytes; human T-lymphocytes have been shown to express a functional RAS and to be able to synthesize Ang II. ${ }^{14,15}$

In our previous study we reported that the hypertensive patients with low-grade inflammation and more marked T-cell RAS activation also had higher body mass index (BMI) and waist circumference values. ${ }^{15}$ This study was aimed at investigating, whether a selective upregulation of circulating lymphocyte-based RAS occurs in subjects with obesity and whether Tcell RAS is modulated differently by Ang II in subjects with obesity with or without low-grade inflammation.

\section{METHODS}

\section{Subjects}

The protocol of this study complies with the principles of the Helsinki declaration. The study was approved by the internal review board and written, informed consent was obtained from each subject.

T-lymphocytes were obtained from 18 subjects with obesity, 43-67 years old, all with BMI >30 who attended our out-patient clinic. No patient was on treatment with angiotensin converting 
enzyme (ACE) inhibitors, AT1 R antagonist or diuretics. Exclusion criteria were both primary and secondary forms of arterial hypertension; ischemic heart disease/acute coronary syndrome or stroke in the previous 6 months; left-ventricular (LV) ejection fraction $<50 \%$; diabetes mellitus; renal failure (creatinine clearance $<60 \mathrm{ml} /$ minute); presence of a major illness such as cancer, liver disease, chronic and acute inflammatory disease or infectious disease in the previous 3 months. These exclusion criteria can induce an activation of systemic and T-cell RAS. No subject was under treatment with anti-inflammatory drugs. Ten subjects without obesity were enrolled into the study as a control group and were comparable for age and sex to the group with obesity. The main demographic and clinical characteristics of enrolled subjects are shown in Table 1. According to high sensitivity C-reactive protein (hsCRP) values at enrolment, subjects with obeisty were divided into 2 groups according to hsCRP value ( $>3 \mathrm{mg} / \mathrm{l}$ or $<3 \mathrm{mg} / \mathrm{l}$ ). Blood hsCRP level and other general chemical tests were carried out as part of a routine medical check-up.

\section{Experimental procedures}

\section{T-lymphocyte isolation}

Venous blood was drawn from the antecubital vein, collected in a sterile flask containing $3.8 \%$ sodium citrate $(9 / 1, \mathrm{vol} / \mathrm{vol})$ and T-lymphocytes isolation was carried out according to our

previous report. ${ }^{14}$ Magnetic beads conjugated to anti-human CD 3 (Miltenyi Biotec, Germany) were used for positive selection of T-lymphocytes. In each incubation experiment mRNA expression of ACE, AT1R and interferon (IFN) gamma, ACE activity and Ang II concentrations were studied in the cell pellet and in the supernatant.

To study the effect of stimulation by Ang II on ACE and AT1R gene expression, ACE activity and Ang II concentrations, T- lymphocytes were cultured in a cell incubator (Forma Scientific) at $37^{\circ} \mathrm{C}$ in a humidified atmosphere at $5 \% \mathrm{CO}_{2}$ for 6,18 and 24 hours with and without $0.05 \mathrm{nmol} / \mathrm{L}$ 
Ang II addition to the culture medium. We used $0.05 \mathrm{nmol} / \mathrm{L}$ of Ang II because this concentration is close to human Ang II plasma values.

Real-Time PCR for ACE gene expression and Reverse Transcriptase-Polymerase Chain Reaction analysis for AT1R and IFN-gamma gene expression

Isolation of total RNA was performed as described previously. ${ }^{14}$ FAM-labelled probes and primers for ACE (ACE Hs00174179_m1, Applied Biosystems, Foster City, CA) and for the housekeeping gene beta-actin (Invitrogen, Milan, Italy) were used. Relative gene expression was calculated as $2^{-\Delta \Delta C t}\left(\Delta C_{t}=C_{t}\right.$ of the target gene minus $C_{t}$ of beta actin). mRNAs for AT1R and IFN gamma were semi-quantified versus the housekeeping gene glyceraldeyde-3-phosphate dehydrogenase (GAPDH) by reverse transcriptase-polymerase chain reaction (RT-PCR)(Applied Biosystems) ${ }^{14}$ using specific primers at annealing temperature and cycle number, as already reported. $^{15}$

\section{Measurement of serum adiponectin}

Human total adiponectin was performed in serum by a commercial kit (R\&D Systems, MN, USA).

Measurement of ACE activity and Ang II concentration in T-cells.

ACE activity measurement and Ang II concentration were performed as previously described. ${ }^{15}$ Ang II levels were expressed as $\mathrm{fmol} / \mathrm{mg}$ of proteins.

\section{Measurement of plasma Ang II concentration}

Plasma Ang II levels were measured by a chemiluminescence immunoassay (CLIA) using a commercial kit (CLIA kit for Angiotensin II, CCA005Hu, Cloud-Clone Corp.) and expressed as pg/ml. 


\section{hsCRP assay}

hsCRP was measured by a commercial kit (Beckman, CA, USA ) with the lowest detection point of $0.1 \mathrm{mg} / \mathrm{l}$.

\section{Statistical Analysis}

Data were reported as frequency (percentage) or mean \pm SD. Between group comparisons were performed with the 1-way analysis of variance (continuous variables) or with the chi-square test (proportions). The time course of both ACE and AT1R gene expression were evaluated within each group: comparisons were performed by means of Student's t-test, paired or unpaired as necessary. Finally, these 2 parameters in each group of subjects with obesity were compared with respective values in the control group using an unpaired t-test.

Probability values of $P<0.05$ were considered significant. All statistical analyses were performed using SPSS, version 17.0 for Windows; SPSS Inc., Chicago, IL, USA statistical software.

\section{RESULTS}

ACE and AT1R gene expression without Ang II stimulation in control subjects and subjects with obesity with or without low grade inflammation.

In control subjects, ACE-mRNA was expressed on T-lymphocytes, and did not modify without Ang II stimulus in the culture medium during the entire incubation period (24 hours) (Figure 1 ). Similarly, T-cell AT1R gene expression did not change in absence of Ang II addition to the culture medium.

All the subjects with obesity, both the groups with hsCRP $<3 \mathrm{mg} / \mathrm{dl}$ and $>3 \mathrm{mg} / \mathrm{dl}$, showed a significantly higher ACE gene expression $(P<0.001)$ than that of the control group when fresh- 
isolated lymphocytes were cultured without Ang II stimulation (Figure 1). The same behavior was found for AT1R gene expression in both groups of subjects with obesity when compared with controls (Figure 2).

\section{Serum adiponectin levels in controls and in subjects with obesity}

Baseline serum adiponectin levels were significantly $(P<0.001)$ higher in control subjects than in subjects with obesity. Data are shown in Figure 4.

\section{Effect of Ang II stimulation on T-cell ACE and AT 1-R gene expression in controls and subjects with obesity}

Addition of $0.05 \mathrm{nmol} / \mathrm{L}$ Ang II significantly increased $(P<0.05)$ ACE mRNA levels in control subjects at 18 and 24 hours of incubation (Figure 1). Similarly, T-cell AT1R gene expression significantly increased $(P<0.005)$ at the $24^{\text {th }}$ hour when T-lymphocytes were stimulated by Ang II (Figure 2).

In subjects with obesity with hsCRP $<3 \mathrm{mg} / \mathrm{dl}$, Ang II stimulation caused a significant early increase in T lymphocyte ACE-mRNA levels at the $6^{\text {th }}$ and $18^{\text {th }}$ hour of incubation $(P<0.005)$, whereas in the group with hsCRP $>3 \mathrm{mg} / \mathrm{dl}$ the significant increase $(P<0.001)$ was observed later, at the $18^{\text {th }}$ hour, and increased further at the $24^{\text {th }}$ hour of incubation; the upregulation of ACEgene expression was significantly higher in the group of subjects with obesity with low-grade inflammation (Figure 1). In both group of subjects with obesity Ang II-stimulation caused an increase in T-cell AT1R mRNA levels at the $6^{\text {th }}$ hour $(P<0.05)$ that was significantly higher than the increase observed in controls; in both groups of subjects with obesity AT1R mRNA levels further increased at the $18^{\text {th }}$ and $24^{\text {th }}$ hour with a peak level at $18^{\text {th }}$ hour $(P<0.001)$ (Figure 2$)$. 


\section{Effect of Ang II stimulation on T-cell IFN-gamma gene expression}

In control subjects and in subjects with obesity, IFN-gamma gene expression was detectable under baseline conditions and did not significantly change during the overall time course when Tlymphocytes were not stimulated by Ang II. Conversely, the addition of Ang II was associated with a significant increase $(P<0.001)$ in IFN-gamma mRNA levels at 18 hours, that peaked at 24-hours without differences between controls and both groups of subjects with obesity (Figure 3).

\section{ACE activity in control subjects and subjects with obesity in basal conditions and after Ang II stimulation.}

Table 2 shows the pattern of ACE activity in the T-cell pellet and supernatant without Ang II stimulation in the 3 groups. In control subjects, T-cell pellet ACE activity was detectable at baseline and did not significantly change at 24 hours; in the supernatant, ACE activity was scarcely detectable during the 24 hours of incubation. At baseline in both groups of subjects with obesity, pellet ACE enzymatic activity was significantly higher $(P<0.001)$ than that assayed in controls, with higher values in subjects with obesity with hsCRP $>3 \mathrm{mg} / \mathrm{dl}$, even if not significantly different in respect to subjects with obesity with hsCRP $<3 \mathrm{mg} / \mathrm{dl}$. During the 24 hours of incubation without Ang II, ACE activity did not significantly change in the pellet, but significantly increased in the supernatant ( $P<0.001$ vs controls). After Ang II stimulation in all subjects with obesity, ACE activity did not significantly change in the pellet, but did significantly increase in the supernatant; the increase in the supernatant was higher in subjects with obesity with hsCRP $>3 \mathrm{mg} / \mathrm{dl}(P<0.001)$ compared with subjects with obesity with hsCRP $<3 \mathrm{mg} / \mathrm{dl}$.

\section{Cell pellet Ang II concentration in control subjects and subjects with obesity in basal conditions and after Ang II stimulation.}


Ang II concentration in the pellet of T-cells of control subjects was significantly lower $(P<$ 0.001 ) than that of subjects with obesity throughout the 24 hours of incubation with and without stimulus. Subjects with obesity with hsCRP $>3 \mathrm{mg} / \mathrm{dl}$ showed a higher Ang II pellet concentration than subjects with obesity with hs CRP $<3 \mathrm{mg} / \mathrm{dl}$; however, at baseline and the $24^{\text {th }}$ hour of incubation without Ang II stimulus, the difference was not statistically significant. With the

addition of Ang II, at the $24^{\text {th }}$ hour pellet Ang II concentration was significantly higher $(P<0.05)$ in subjects with obesity with hsCRP >3 mg/dl (Table 2)

\section{Ang II plasma concentration in control subjects and subjects with obesity}

Ang II levels in plasma of control subjects and subjects with obesity did not significantly differ among the groups studied $(12.5 \pm 4.5 \mathrm{pg} / \mathrm{ml}, 11.6 \pm 5.2 \mathrm{pg} / \mathrm{ml}, 13.1 \pm 6.2 \mathrm{pg} / \mathrm{ml}$ in controls, subjects with obesity with hsCRP $<3$ and subjects with obesity with hsCRP $>3$, respectively).

\section{DISCUSSION}

Our data show a selective upregulation of circulating lymphocytic RAS in subjects with obesity, independent of low-grade inflammation that amplified the upregulation of T-cell RAS by Ang II in subjects with obesity with hsCRP $>3 \mathrm{mg} / \mathrm{dl}$.

ACE and AT1R gene expression of T-cells in subjects with obesity was significantly higher than in controls and further increased after Ang II stimulation of T-cells. Subjects with obesity with low grade inflammation showed a higher prevalence of metabolic syndrome. The upregulation of ACEgene expression was significantly higher in the group of subjects with obesity with low-grade 
inflammation and was associated with a significant release of ACE activity in the supernatant of Tcells; remarkably, the addition of Ang II stimulus to cultured T-cells caused the almost complete release of ACE activity in the medium.

Subjects with obesity with hsCRP> 3mg/dl showed a significantly higher $(P<0.05) \mathrm{T}$ - cell Ang II pellet concentration with Ang II stimulation. The increase of Ang II concentration in activated Tcells could have clinical relevance when occurring within tissues where circulating T-cells are known to migrate. The enhanced Ang II release by T-cells could start a dangerous positive loop in inflamed tissues and further amplify the activation of the immune response. ${ }^{6}$

Remarkably, our in vitro finding of the marked release of ACE enzymatic activity in the supernatant by cultured T-cell from subjects with obesity with low grade inflammation, in vivo could give rise to a local increased activation of other cells endowed with AT1R, such as monocyte and macrophage infiltration in subjects with obesity and other tissues by the de novo synthesized Ang II. ${ }^{2}$

It is well-known that obesity predisposes to metabolic disease and that many type 2 diabetes patients are obese. Several studies in humans and animals find obesity associated with enhanced activity of both systemic RAS and adipose tissue RAS. ${ }^{10}$ In particular, AGT synthesis is very developed in adipocytes and contributes significantly to the systemic pool. The activity of tissue RAS is higher in visceral/central adipose tissue than in subcutaneous tissue and this finding might explain the risks related to upper body visceral fat accumulation. ${ }^{16}$ Many immediate effects of Ang II are mediated via the AT1R through classical G-protein-dependent signaling pathways. Stimulation of the AT1R also activates the membrane-bound $N A D(P) H$ oxidase leading to increased generation of reactive oxygen species (ROS). The $\mathrm{NAD}(\mathrm{P}) \mathrm{H}$ formed ROS may activate mitochondrial $\mathrm{K}_{\text {ATP }}$ channels to additionally burst mitochondrial ROS generation. ${ }^{17}$ After its generation, intracellular ROS can activate many down-stream molecules, such as mitogen-activated protein 
kinases, protein tyrosine phosphatases, protein tyrosine kinases, and transcriptional factors, which, if chronically activated, will promote inflammation, atherosclerosis, thrombosis, and fibrogenesis. ${ }^{18,19,20}$

Low-grade inflammation has been widely associated with atherosclerosis and cardiovascular disease. CRP directly binds highly atherogenic oxidized low-density lipoprotein cholesterol (LDL-C) and is present within lipid-laden plaques; CRP may facilitate lympho-monocyte adhesion and transmigration into the vessel wall, a critical early step in the atherosclerotic process. ${ }^{21}$

In our previous study we demonstrated that there is an increase in circulating T-lymphocyte RAS activation in hypertensive patients, related to low-grade inflammation (increased serum hsCRP) and BMI. ${ }^{14}$ Hypertensive patients with low-grade inflammation presented upregulation of lymphocytic RAS and showed the highest left ventricular mass index (LVMI) and renal resistive index values, making it possible to hypothesize that in patients with low-grade inflammation higher local cardiac and renal Ang II concentration due to T-cell RAS activation could facilitate a worse progression of hypertension-related tissue organ damage.

Circulating T-lymphocytes RAS activation in subjects with obesity could be involved in organ damage, as seen for hypertensive subjects. It is well-known that adipose tissue (particularly visceral adipose tissue) of obese rodents presents a major reserve of CD4+ and CD8+ Tlymphocytes, compared to lean rodents. ${ }^{22}$ T-lymphocytes could play an important role also in metabolic and vascular complications related to obesity, as they modulate immune response in atherogenesis producing Th1 cytokines and promoting classically activated M1 macrophages. ${ }^{23}$

The low number of subjects investigated, weaken our results, even if the homogenous pattern of lymphocyte RAS in subjects with obesity with or without low grade inflammation strongly supports our findings. T-cell RAS activation was studied by the measurement of ACE and AT1R gene expression without the dosage of renin, angiotensinogen and 
AT1R proteins, because ACE and AT1R are the key steps of RAS activity. Remarkably, ACE and AT1R are the target of drugs. The lack of a follow-up limits the understanding of potential in vivo tissue damage due to RAS lymphocyte-based activation and due to the increased local concentration of Ang II shown in vitro.

In conclusion, low grade inflammation amplifies angiotensin II activation of upregulated circulating T-cell renin angiotensin system in subjects with obesity. In individuals with obesity the enhanced Ang II release by T-cells in the presence of low grade inflammation could start a dangerous positive loop in inflamed tissues and further amplify the activation of the immune response.

\section{Author Contributions}

M.Coppo and M.Boddi conceived the work, L.Poggesi enrolled the subjects and performed the clinical evaluation, M.Coppo and M.Bandinelli performed the dosages, M.Chiostri performed the statistical evaluation, M.Boddi and M.Coppo wrote the manuscript. 


\section{REFERENCES}

1.Lastra G, Sowers JR. Obesity and cardiovascular disease: role of adipose tissue, inflammation, and the renin-angiotensin-aldosterone system. Horm Mol Biol Clin Investig. 2013 Sep;15(2):49-57. doi: $10.1515 / \mathrm{hmbci}-2013-0025$

2.Ramalingam $\mathrm{L}$, Menikdiwela $\mathrm{K}$, LeMieux $\mathrm{M}$, et al. The renin angiotensin system, oxidative stress and mitochondrial function in obesity and insulin resistance. Biochim Biophys Acta. 2017 May;1863(5):1106-1114. doi: 10.1016/j.bbadis.2016.07.019.

3.Chawla A, Nguyen KD, Goh YP. Macrophage mediated inflammation in metabolic disease. Nat Rev Immunol. 11(11):738-749.

4.Ouchi N, Parker JL, Lugus JJ, et al. Adipokines in inflammation and metabolic disease. Nat Rev Immunol. 2011; Feb; 11(2):85-97.

5.Borghi F, Sevà-Pessoa B, Grassi-Kassisse DM. The adipose tissue and the involvement of the renin-angiotensin-aldosterone system in cardiometabolic syndrome. Cell Tissue Res. 2016 Dec;366(3):543-548.

6.Kalupahana NS, Massiera F, Quignard-Boulange A et al. Overproduction of angiotensinogen from adipose tissue induces adipose inflammation, glucose intolerance, and insulin resistance. Obesity (Silver Spring) 2012 Jan;20(1):48-56. doi:10.1038/oby.2011.299.

7. Iantorno M, Campia U, Di Daniele N, et al. Obesity, inflammation and endothelial dysfunction. J Biol Regul Homeost Agents. 2014 Apr-Jun;28(2):169-76.

8. A. Feraco, A. Armani, C. Mammi, et al. Role of mineralocorticoid receptor and reninangiotensin-aldosterone system in adipocyte dysfunction and obesity. J. Steroid Biochem. Mol. Biol. 137 (0) (2013) 99-106. 
9. Kim S, Soltani Bejnood M, Quignard Boulange A, et al. The adipose renin angiotensin system modulates systemic markers of insulin sensitivity and activates the intrarenal renin angiotensin system. J Biochem Biotechnol . 2006(5):27012.

10.Kalupahana NS, Massiera F, Quignard-Boulange A, et al. Overproduction of angiotensinogen from adipose tissue induces adipose inflammation, glucose intolerance and insulin resistance. Obesity $2012 ; 20(1): 48-56$.

11.Maury E, Brichard SM. Adipokine dysregulation, adipose tissue inflammation and metabolic syndrome. Mol Cell Endocrinol. 2010 Jan 15; 314(1):1-16.

12.Jing F, Mogi M, Horiuchi. Role of renin-angiotensin-aldosterone system in adipose tissue dysfunction. Mol Cell Endocrinol. 2013 Sep 25; 378(1-2):23-8.

13. Maeda A, Tamura K, Wakui H, et al. Effects of Ang II Receptor Blocker Irbesartan on Adipose Tissue Function in Mice with Metabolic Disorders. J Med Sci. 2014; 11(6): 646-651. doi: 10.7150/ijms.8577PMCID: PMC4021097

14.Coppo M, Bandinelli M, Berni A, et al. Ang II up-regulation of the T-lymphocyte reninangiotensin system is amplified by low-grade inflammation in human hypertension. Am J Hypertens. 2011 Jun;24(6):716-2.

15.Coppo M, Boddi M, Bandinelli $\mathrm{M}$, et al. Angiotensin II upregulates renin-angiotensin system in human isolated T lymphocytes. Regul Pept. 2008 Nov 29;151(1-3):1-6. doi:

10.1016/j.regpep.2008.07.010.

16. Bastien M, Poirier P, Lemieux I, et al . Overview of epidemiology and contribution of obesity to cardiovascular disease. JP Prog Cardiovasc Dis. 2014 Jan-Feb; 56(4):369-81.

17.Zhang GX, Lu XM, Kimura S, et al. Role of mitochondria in angiotensin II-induced reactive oxygen species and mitogen-activated protein kinase activation. Cardiovasc Res. 2007 Nov 1; 76(2):204-12. 
18.Mehta PK, Griendling KK . Angiotensin II cell signaling: physiological and pathological effects in the cardiovascular system. Am J Physiol Cell Physiol. 2007 Jan; 292(1):C82-97.

19.Yousuf O, Mohanty BD, Martin SS, et al. High-sensitivity C-reactive protein and cardiovascular disease: a resolute belief or an elusive link? J Am Coll Cardiol. 2013 Jul 30;62(5):397-408. doi: 10.1016/j.jacc. 2013.05.016. Epub 2013 May 30.

20.Reaven GM. The insulin resistance syndrome: definition and dietary approaches to treatment. Annu Rev Nutr. 2205; 25(4): 391-406.

21.Romeo GR, Lee J, Shoelson SE. Metabolic syndrome, insulin resistance and roles of inflammation - mechanisms and therapeutic targets. Arterioscler Thromb Vasc Biol. 2012; 32(8):1771-6.

22.Libby P, Okamoto Y, Rocha VZ, et al. Inflammation in atherosclerosis: transition from theory to practice. Circ J. 2010 Feb;74(2):213-20. Epub 2010 Jan 9.

23.Rocha VZ, Libby P. Obesity, inflammation and atherosclerosis. Nat Rev Cardiol. 2009 Jun;6(6):399-409. doi: 10.1038/nrcardio.2009.55. Epub 2009 Apr 28. 


\section{Figure Legend}

Figure 1. ACE gene expression in control and subjects with obesity.

Abbreviations: Ang II, angiotensin II; hsCRP, high sensitivity C-reactive protein.

${ }^{\circ} P<0.001$ vs controls (unpaired t-test).

${ }^{*} P<0.05$ vs without stimulus (paired t-test).

$\# P<0.001$ vs without stimulus (paired t-test).

Figure 2. AT1R gene expression in control and subjects with obesity.

Abbreviations: Ang II, angiotensin II; hsCRP, high sensitivity C-reactive protein.

${ }^{\circ} P<0.05$ vs controls.

$\S P<0.001$ vs controls; ${ }^{*} P<0.005$ vs without stimulus.

$\# P<0.001$ vs without stimulus.

Figure 3. IFNgamma gene expression in control and subjects with obesity.

Abbreviations: Ang II, angiotensin II; hsCRP, high sensitivity C-reactive protein. $\# P<0.001$ vs without stimulus.

Figure 4. Adiponectin serum concentration in control and subjects with obesity.

Abbreviations: hsCRP, high sensitivity C-reactive protein.

$\# P<0.001$ vs controls. 
